\title{
Productivity analysis of an electronics re- manufacturing system through stochastic Petri nets and artificial neural networks
}

\author{
Mehmet Ali Arslan ${ }^{1}$ and Ismail Fidan ${ }^{2 \star}$ \\ ${ }^{1}$ Department of Mechanical Engineering, Gebze Institute of Technology, P. K. 141 Gebze, Kocaeli 41400, Turkey. \\ ${ }^{2}$ Department of Manufacturing and Industrial Technology, College of Engineering, Tennessee Technological University, \\ P. O. Box 5003 Cookville, TN 38505, USA. \\ Accepted 1 April, 2011
}

This paper provides Petri net (PN) modeling and performance analysis of a surface mount device (SMD) electronics manufacturing assembly line for an automated remanufacturing of printed circuit boards. Concentrating on the operational aspects, PN models for an automated assembly stations were constructed. These models enable designers to have a better understanding of the system control and analysis from the graphical representations of PNs. In this context, the selection of the particular buffer size and its effects on the production rate of the transferline are explored. PN models are designed to analyze two different transferlines and to find out when local gains propagate to the end of the transferline. Furthermore, artificial neural networks (ANN) are proposed as a fast function approximation tool for a rapid re-analysis of the remanufacturing system. ANN can easily predict the output of the transferline for unknown input patterns when the input and output relation is monotonically increasing or decreasing. This capability of the ANN proves to be useful to analyze the transferline when there is no further information available. The approaches as presented in this paper can be generalized and applied to many other applications of multi-robot assembly systems.

Key words: Electronics remanufacturing, stochastic Petri nets, artificial neural networks, surface mount device, performance analysis.

\section{INTRODUCTION}

In the last few years, the number of rework stations available on the electronics manufacturing market has grown considerably including automated ones, but there has still been no significant reduction in the number of defects. In electronics manufacturing, rework is defined as the activity that replaces defective components with those that are acceptable, such that the populated board performs to specifications. Increasing product complexity, decreasing component size, and using double-sided boards have made rework more difficult and the economic reworking of printed circuit board assemblies is

${ }^{*}$ Corresponding author. E-mail: i.fidan@ieee.org. Tel: (931) 372-6298. Fax: (931) 372-3813. one of the main problems facing electronics manufacturers. Printed circuit board assembly manufacturing has been relatively improved with fully automated, accurate assembly machines and the use of robots (Dariavach et al., 2010).

Although significant improvement in automated rework has also been made by the authors, it has been shown the outcome of the automated rework line has not produced a high enough reliable yield percentage (Fidan et al., 2004; Fidan, 2004; Fidan et al., 2006). The objective of this investigation is to make a contribution towards this surface mount electronics remanufacturing process by analyzing their performance via stochastic Petri nets and artificial neural networks, so that the defects that necessitate a rework operation could be analyzed to predict the production rate of the transferline, 


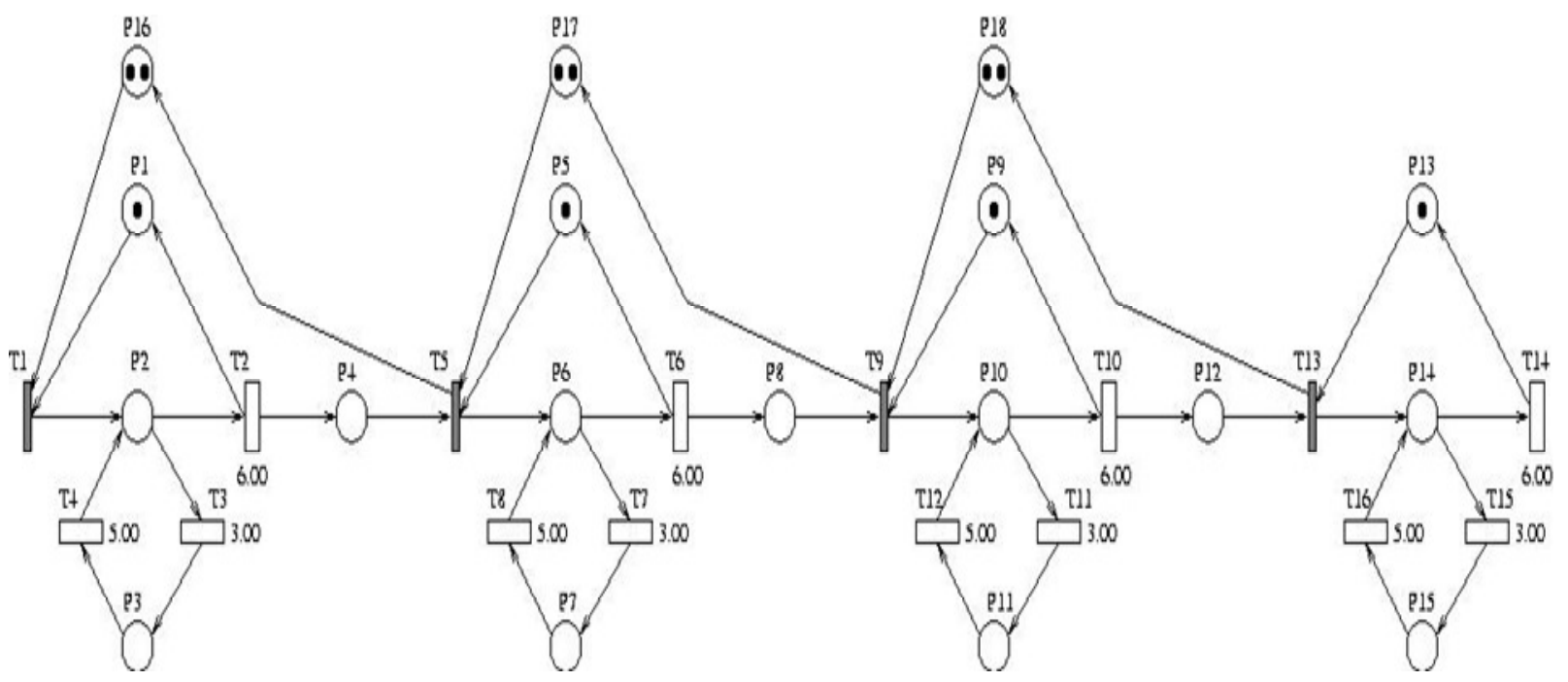

Figure 1. PN Model of the SMD assembly station for case 1.

by using prior information instead of performing reanalysis of the whole system. There is a growing interest in automating electronic assembly lines in order to compete effectively in today's emerging market place. The overall goal of the research in electronic manufacturing automation is to offer solutions to achieve higher speeds and more flexibility. Manufacturing productivity can be inreased further by studying the optimal settings of operation, including sequence of component placements, and planning of assembly task (Vendan and Sakthidhasan., 2010). PNs can be used to study the problem of optimal operational settings by concurrent assembly systems and to evaluate the production rates of various possible models.

The whole problem of modeling and control of manufacturing systems has its roots in discrete-event dynamic systems. In a manufacturing system, machine or robot failures and repairs, sudden changes in demand, and cell blockage or starvation are all events occurring at discrete instants. The initiation or termination of these events can propagate from cell to cell affecting numerous performance measures, e.g. buffer size vs. production rate, deadlock, etc. Petri nets have evolved into a powerful tool for analyzing asynchronous concurrent systems and have been shown to be useful in modeling flexible manufacturing systems (Al-Jaar and Desrochers., 1989; DuBois and Stecke., 1982; Desrochers, 1990). They can provide accurate models because, Petri nets can capture the precedence relations and structural interactions of concurrent and asynchronous events. Deadlock, conflicts and buffer size can be modeled easily. Petri net models have a well developed mathematics formulation that allows a qualitative and quantitative analysis of the system.

In this paper, we present the PN method to model and analyze a Surface Mount Device (SMD) assembly system for remanufacturing printed circuit boards. The paper is

mainly organized as follows:

1. The effects of the varying buffer size from its nominal value.

2. The level of an increase in local production rate at the preceding cell.

3 . The time this local production gain propagates to the end of the transferline resulting in a gain.

4. The use of ANN as a fast function approximation tool for rapid reanalysis of the SMD remanufacturing station.

\section{MATERIALS AND METHODS}

A flexible robotic assembly station for printed circuit boards' remanufactuiring includes the following components:

Cell 1 - Machine 1, solder paste is dispensed onto the board.

Cell 2 - Robot 1, component is picked up and placed onto dispensed board.

Cell 3 - Robot 2, component is reflowed by using laser energy.

Cell 4 - Machine 2, complete board is inspected before it is used.

Such a rework system which is aimed at remanufacturing SMDs, is considered in this study. $P$ represents the overall production rate at which parts emerge from the transferline. Two cases examined and all analysis are performed by using SPNP (stochastic Petri net's package) software. Basic knowledge of PNs and related modeling issues can be found in many references such as DuBois and Stecke. (1982), Desrochers (1990) and Rosenblatt (1962).

\section{Case 1}

The cells (robots or machines) are identical with examples of rates given subsequently. A part cannot enter a cell unless the cell's buffer has a vacancy. The live and bounded PN model in Figure 1, is developed to calculate overall production rate of the transferline. 


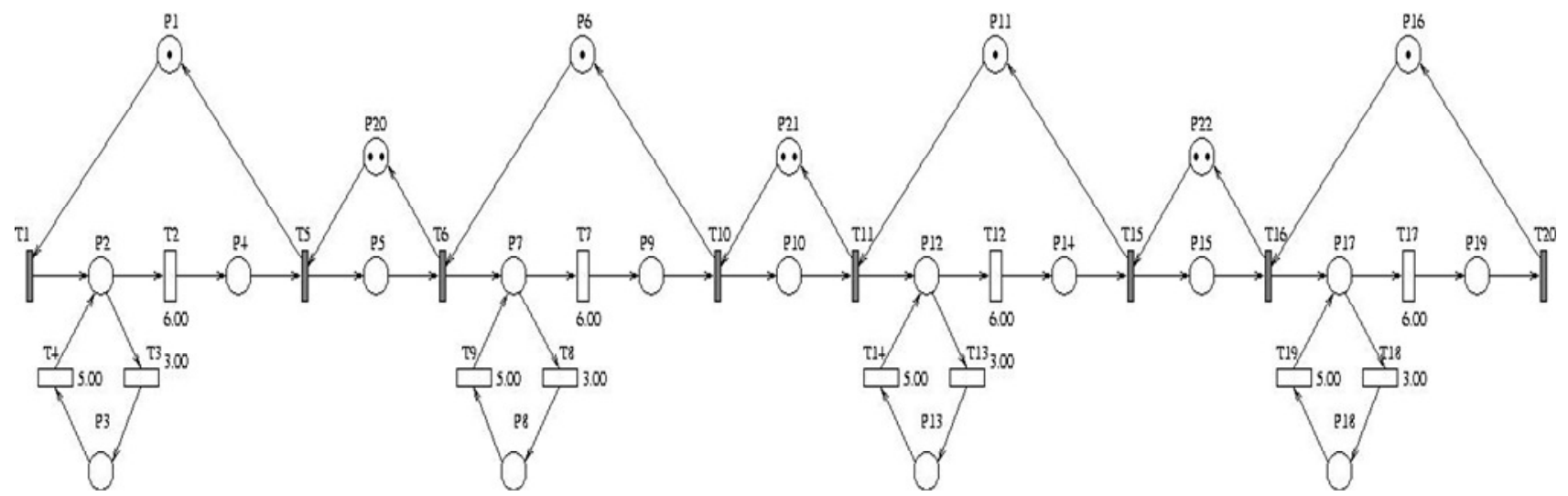

Figure 2. PN Model of the SMD assembly station for case 2.

The number of tokens in the randomly chosen buffer 2 and 3 is varied from 1 to 5 to calculate an increase on the transferline production rate vs. varying buffer size at each underlying buffer location.

\section{Case 2}

A part can enter a cell even if the cell's buffer is full, but cannot exit the cell until there is a vacancy. This PN model is presented in Figure 2. The same steps are followed as explained in Case 1 for buffer $\mathrm{N}_{3}$.

\section{Assumptions}

Modeling automated manufacturing systems inherently leads to models with a large and complicated number of states. In this study, the following assumptions are made for simplification:

1. Processing, failure and repair time are exponentially distributed

2. Failures occur when cells are operating

3. The first cell never starved and the last cell is never blocked

Problem parameters for related units,

$t_{p}$ (part processing rate $)=6 \%$

$t_{f}$ (machine or robot failure rate) $=3 \%$

$t_{r}$ (machine or robot repair rate $)=5 \%$

for Machines, $M_{1}, M_{2}, M_{3}, M_{4}$.

$N$ (buffer size $)=2$ for buffers, $B_{1}, B_{2}, B_{3}$.

\section{Analytical Investigation}

Under the aforementioned assumptions both cases are first designed then analyzed using SPNP software as presented in the following.

\section{Case 1}

For case 1, $P$ and $T$ invariants are found to be as follows:

1. $m\left(p_{1}\right)+m\left(p_{2}\right)+m\left(p_{3}\right)=1$

2. $m\left(p_{2}\right)+m\left(p_{3}\right)+m\left(p_{4}\right)+m\left(p_{16}\right)=k=2$

3. $m\left(p_{5}\right)+m\left(p_{6}\right)+m\left(p_{7}\right)=1$
4. $m\left(p_{6}\right)+m\left(p_{7}\right)+m\left(p_{8}\right)+m\left(p_{17}\right)=k=2$

5. $m\left(p_{9}\right)+m\left(p_{10}\right)+m\left(p_{11}\right)=1$

6. $m\left(p_{10}\right)+m\left(p_{11}\right)+m\left(p_{12}\right)+m\left(p_{18}\right)=k=2$

7. $m\left(p_{13}\right)+m\left(p_{14}\right)+m\left(p_{15}\right)=1$

and,

1. $y_{1}=\left\{t_{3}, t_{4}\right\}$

2. $\mathrm{y}_{2}=\left\{\mathrm{t}_{7}, \mathrm{t}_{8}\right\}$

3. $\mathrm{y}_{3}=\left\{\mathrm{t}_{11}, \mathrm{t}_{12}\right\}$

4. $y_{4}=\left\{t_{15}, t_{16}\right\}$

5. $y_{5}=\left\{t_{1}, t_{2}, t_{5}, t_{6}, t_{9}, t_{10}, t_{13}, t_{14}\right\}$

The invariants $1,3,5,7$ relate the activities at cell 1,2,3,4 indicating each cell can be up and free, busy or down, or being repaired. The invariants 2,4,6 point out that, the total number of parts in the buffers and in the cells are always equal to $k=2$. The invariants, $T_{1}, T_{2}, T_{3}, T_{4}$ represent failure and repair cycles, $T_{5}$ represents a production cycle. One can easily see and interpret from the $T$ invariants that, all of the transitions (at least one of them) can be fired, for any reachable marking. Hence, there exist no deadlock. As a result, it can be concluded that the underlying PN is live, and since every place in the PN model is covered by $P$-invariant, it is also bounded. SMD part remanufacturing rate of the system is:

$P=\operatorname{rate}\left(\mathrm{t}_{14}\right) \times \operatorname{prob}\left(\mathrm{m}\left(\mathrm{p}_{14}\right)\right)=2.124$ parts $/ \mathrm{h}$

By changing the number of tokens in buffers 2 and 3 from 1 to 5 , the following gradient distributions seen in Table 1 were found.

\section{Case 2}

After analysis of case 2, P and T invariants were found to be as follows:

1. $m\left(p_{5}\right)+m\left(p_{20}\right)=k=2$

2. $m\left(p_{10}\right)+m\left(p_{21}\right)=k=2$

3. $m\left(p_{15}\right)+m\left(p_{22}\right)=k=2$

4. $m\left(p_{1}\right)+m\left(p_{2}\right)+m\left(p_{3}\right)+m\left(p_{4}\right)=k=1$

5. $m\left(p_{6}\right)+m\left(p_{7}\right)+m\left(p_{8}\right)+m\left(p_{9}\right)=1$

6. $m\left(p_{11}\right)+m\left(p_{12}\right)+m\left(p_{13}\right)+m\left(p_{14}\right)=1$

7. $m\left(p_{16}\right)+m\left(p_{17}\right)+m\left(p_{18}\right)+m\left(p_{19}\right)=1$

and

1. $\mathrm{y}_{1}=\left\{\mathrm{t}_{3}, \mathrm{t}_{4}\right\}$ 
Table 1. The reflections of the changes in buffers 2 and 3.

\begin{tabular}{ccc}
\hline $\boldsymbol{P}_{\boldsymbol{i + 1}}-\boldsymbol{P}_{\boldsymbol{i}}$ & $\boldsymbol{\Delta}_{\boldsymbol{N}}$ in $\boldsymbol{N}_{\mathbf{2}}$ & $\boldsymbol{\Delta P}_{\boldsymbol{P}} / \boldsymbol{\Delta}_{\boldsymbol{N}}$ \\
\hline $2.12-1.96$ & $N_{2}=1$ to 2 & 0.155 \\
$2.21-2.12$ & $N_{2}=2$ to 3 & 0.094 \\
$2.28-2.21$ & $N_{2}=3$ to 4 & 0.064 \\
$2.33-2.28$ & $N_{2}=4$ to 5 & 0.047 \\
& & \\
& & \\
$\boldsymbol{P}_{\boldsymbol{i + 1} \mathbf{1}}-\boldsymbol{P}_{\boldsymbol{i}}$ & $\boldsymbol{\Delta}_{\boldsymbol{N}}$ in $\boldsymbol{N}_{\mathbf{3}}$ & $\boldsymbol{\Delta}_{\boldsymbol{P}} / \boldsymbol{\Delta}_{\boldsymbol{N}}$ \\
$2.12-1.99$ & $N_{3}=1$ to 2 & 0.129 \\
$2.19-2.12$ & $N_{3}=2$ to 3 & 0.068 \\
$2.23-2.19$ & $N_{3}=3$ to 4 & 0.039 \\
$2.25-2.23$ & $N_{3}=4$ to 5 & 0.023 \\
\hline
\end{tabular}

$\Delta_{N}=$ Change in the number of tokens; $\Delta_{P}=$ Change in the overall production rate; $\mathrm{P}=$ production rate $($ parts $/ \mathrm{h})$

Table 2. The reflection of the changes in buffer 3 .

\begin{tabular}{ccc}
\hline $\boldsymbol{P}_{\boldsymbol{i + \boldsymbol { 1 }}}-\boldsymbol{P}_{\boldsymbol{i}}$ & $\boldsymbol{\Delta}_{\boldsymbol{N}}$ in $\boldsymbol{N}_{\boldsymbol{3}}$ & $\boldsymbol{\Delta} \boldsymbol{P} / \boldsymbol{\Delta}_{\boldsymbol{N}}$ \\
\hline $2.37-2.28$ & $N_{3}=1$ to 2 & 0.083 \\
$2.42-2.37$ & $N_{3}=2$ to 3 & 0.050 \\
$2.45-2.42$ & $N_{3}=3$ to 4 & 0.032 \\
$2.47-2.45$ & $N_{3}=4$ to 5 & 0.021 \\
\hline
\end{tabular}

2. $y_{2}=\left\{t_{8}, t_{9}\right\}$

3. $\mathrm{y}_{3}=\left\{\mathrm{t}_{13}, \mathrm{t}_{14}\right\}$

4. $\mathrm{y}_{4}=\left\{\mathrm{t}_{18}, \mathrm{t}_{19}\right\}$

5. $\mathrm{y}_{5}=\left\{\mathrm{t}_{1}, \mathrm{t}_{2}, \mathrm{t}_{5}, \mathrm{t}_{6}, \mathrm{t}_{7}, \mathrm{t}_{10}, \mathrm{t}_{11}, \mathrm{t}_{12}, \mathrm{t}_{15}, \mathrm{t}_{16}, \mathrm{t}_{17}, \mathrm{t}_{20}\right\}$

One can make similar conclusions as in case 1. The underlying PN model is live and bounded. Production rate of the system is:

$P=\operatorname{rate}\left(\mathrm{t}_{17}\right) \times \operatorname{prob}\left(\mathrm{m}\left(\mathrm{p}_{17}\right)\right)=2.372$ parts $/ \mathrm{h}$.

By changing the number of tokens only in buffer 3 from 1 to 5 , the following gradient distributions seen in Table 2 were found. In cases 1 and 2, as presented in Figures 3 and 4, after increasing a buffer size from the nominal value of $N_{3}$, an increase in (local) production rate occurred at the preceding cells. This production gain propagated to the end of the transferline by increasing $N_{2}$ and $N_{3}$ buffer sizes from their nominal value. All the curves tend to saturate at a certain buffer size. Therefore, it is useless to use huge buffer sizes which only increases the inventory. At this point it can be concluded that there is no point in increasing buffer size because it reaches almost its optimal value. If both curves are matched for case 1, one can easily see that by increasing the buffer size $N_{2}$, overall production rate increases much more than by increasing buffer size $N_{3}$. This can be interpreted that an increase in buffer $N_{2}$ gives better overall production rate in comparison with $N_{3}$. Local production gain in cell $i$ may not propagate through the line and does not result in a change in line production rate if the last cell in the transferline has an extremely high failure rate.

In case 2, an increase in buffer size $N_{2}$ has resulted in an increase in the (local) production rate at the preceding cells. This production gain can be propagated to the end of the transferline by increasing only $N_{3}$ for this case. The slope of the curve tends to decrease and saturate at an optimal buffer size. The overall production rate is $10 \%$ higher than case 1 .

\section{Using artificial neural networks in the analysis}

The multilayer perceptron architecture (Hajela et al., 1992) of ANN has been proposed for using a trained network as a rapid reanalysis tool (Berke and Hajela., 1992; Fu et al., 1992; Arslan, 1993). Briefly, a trained network is obtained as a two-step process and is initiated with the definition of the network architecture, including the number of input-output neurons, the number of hidden layers, and the neurons in each of these layers. A set of input patterns considered to be representative of the problem of interest, are presented to the network input nodes; the output corresponding to each of these patterns is obtained at the output layer neurons as a function of interconnection weights and bias constants.

The steepest descent method was used to minimize the difference between the network predicted and known outputs by adjusting weights and bias constants by a modified delta rule. When the network error is below some threshold value, the network is considered to be trained and can be used to predict outputs for input patterns which are not presented to the network before. This method is also known as back-propagation learning algorithm. An example of a neural network model is shown in Figure 5. The prediction capability of neural network allows one to perform rapid reanalysis of the transferline in terms of buffer sizes and production rate. This paper presents this approach by implementing to case 1 , in which $N_{2}$ buffer size varies from 1 to 5 .

\section{RESULTS AND DISCUSSION}

The relations between three independent variables which are $N_{1}, N_{2}$ and $N_{3}$ buffer sizes were chosen as the input parameters and overall production rate $P$ was chosen as an output parameter. During training a network with 3 neurons in the input layer, 7 neurons in the hidden layer, and 1 neuron in the output layer (denoted by $3^{\star} 7^{\star} 1$ ) was trained with 10 input/output patterns. The network error for the mapping between $x_{i}$ (inputs) and $y_{j}$ (outputs) was reduced to less than $1 \%$ as it can be seen in Table 3 .

After training, generalization capability of the net was tested by using two existing buffer size information. Furthermore, in case 1, two more new input patterns were presented to the network, of which actual outputs are not known from SPNP analysis of the system. Predictions of the artificial neural net which are obtained are consistent with the previous results and new gradients tend to decrease. The ANN and SPNP analysis results for buffer $\mathrm{N}_{2}$ can be seen in Figure 6, where we can see that ANN is useful in approximating the system production rate. This capability of the ANN may save a lot of time and money for quick estimations. The results are summarized in the following table.

\section{Conclusions}

In this study, PN modeling approach for the design of flexible rework systems for remanufacturing of SMDs was presented. PN models have been constructed for an 


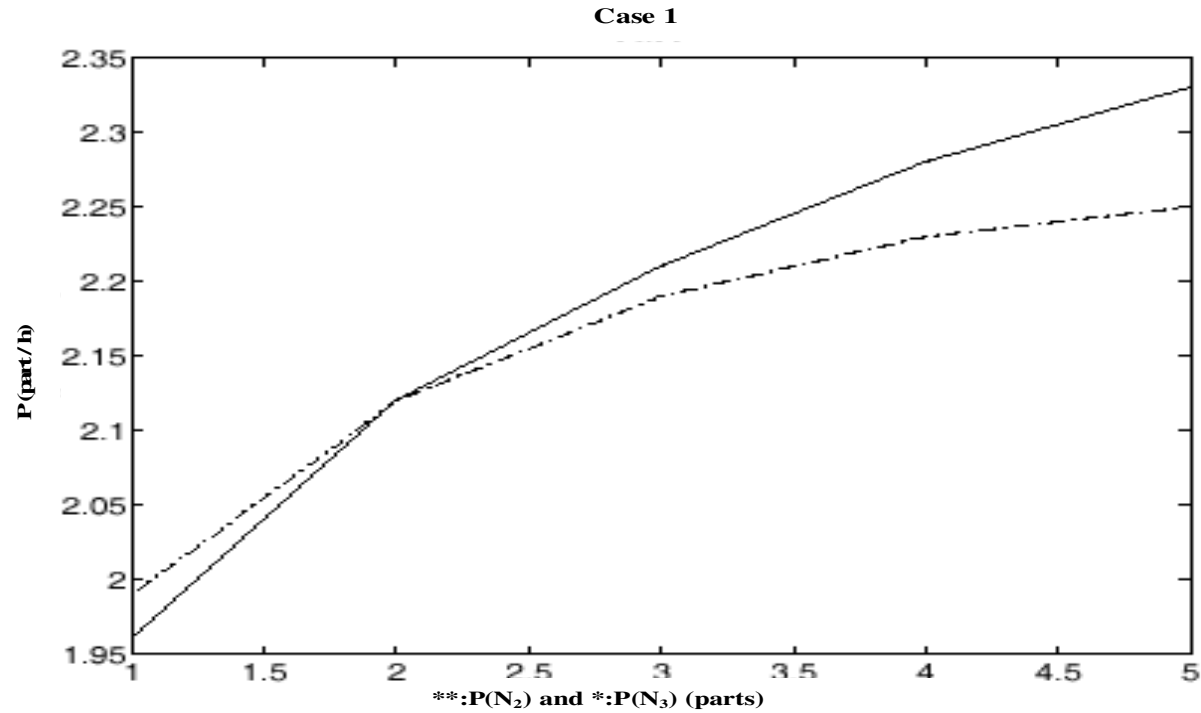

Figure 3. Average production rate vs. $\mathrm{N}_{2}$ and $\mathrm{N}_{3}$ for case 1 .

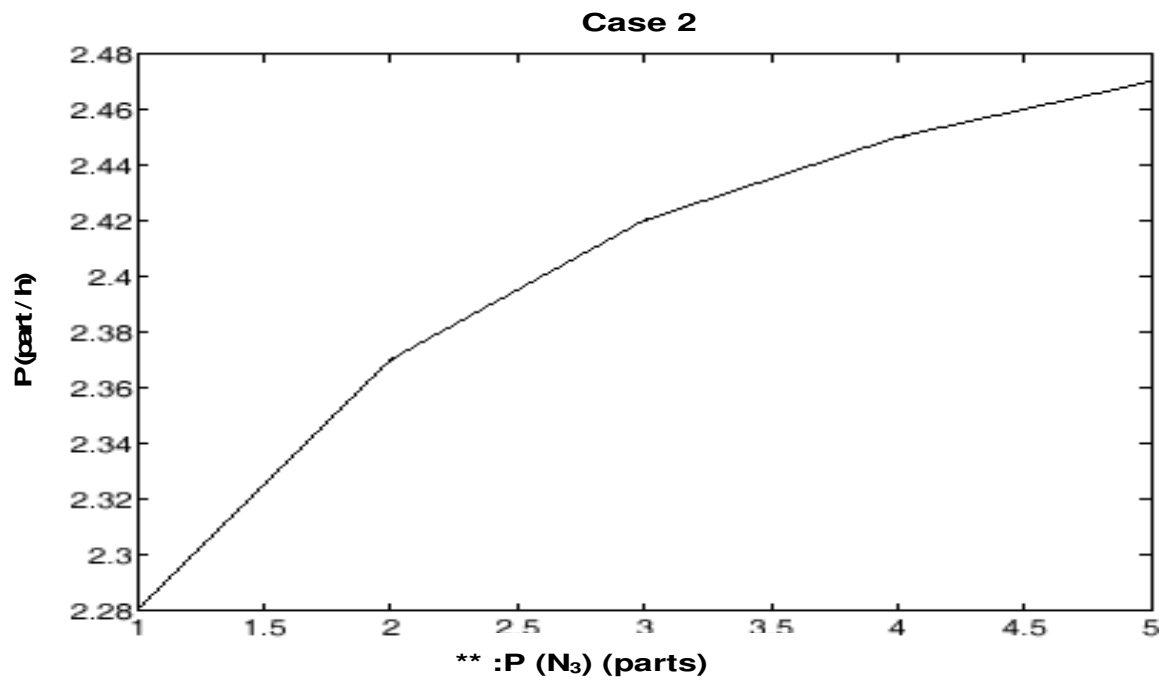

Figure 4. Average production rate vs. $\mathrm{N}_{3}$ for case 2 .

Table 3. Relative error between the actual and predicted results.

\begin{tabular}{cccc}
\hline $\boldsymbol{N}_{\mathbf{1}} \cdot \boldsymbol{N}_{\mathbf{2}} \cdot \boldsymbol{N}_{\mathbf{3}}$ & Actual & Predicted & Relative error \\
\hline $2-5-2$ & 2.330 & 2.323 & 0.003 \\
$2-6-2$ & $\mathrm{~N} / \mathrm{A}$ & 2.338 & $\mathrm{~N} / \mathrm{A}$ \\
$2-7-2$ & $\mathrm{~N} / \mathrm{A}$ & 2.346 & $\mathrm{~N} / \mathrm{A}$ \\
$2-2-5$ & 2.255 & 2.276 & 0.009 \\
$2-2-6$ & $\mathrm{~N} / \mathrm{A}$ & 2.306 & $\mathrm{~N} / \mathrm{A}$ \\
$2-2-7$ & $\mathrm{~N} / \mathrm{A}$ & 2.322 & $\mathrm{~N} / \mathrm{A}$ \\
\hline
\end{tabular}

existing flexible station. As a step toward the design of a flexible SMD assembly system, PN models help the designer understand graphically the system operations and characteristics. The system performance has been evaluated for two cases. The results indicate that case 2 exhibits both higher system throughput and better robot and machine utilization than the corresponding marked graph.

Consequently, according to the ratio between an increase of transferline production and buffer size, one can allocate the buffer size at each location to maximize for performance index. As seen in cases 1 and 2, after increasing buffer sizes $N_{2}$ and $N_{3}$, overall production rate increases to a certain degree after a certain buffer size, no increase can be observed. In case 1 , an increase in buffer $\mathrm{N}_{2}$ has higher effect on production rate than buffer $N_{3}$. Finally, ANN method has been proven to be very 


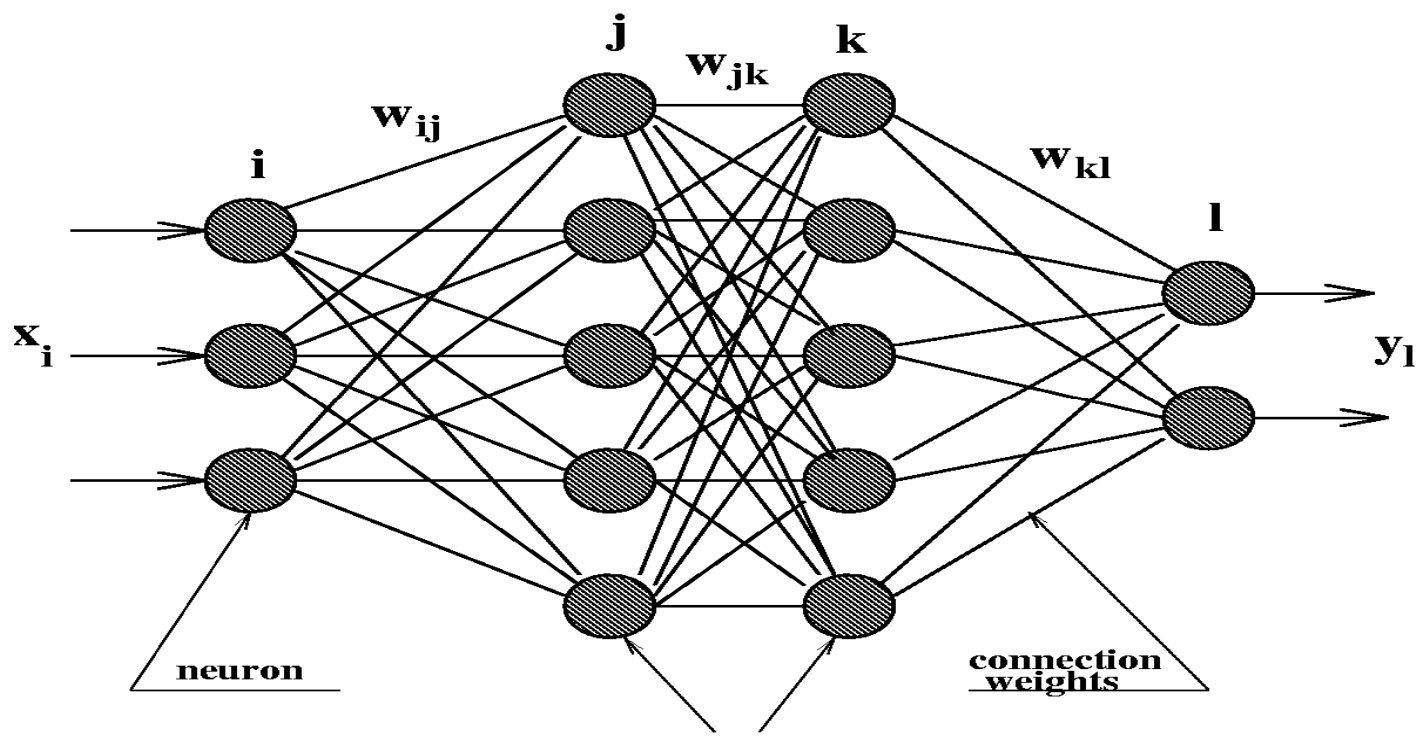

Input layer

Hidden layers

Output layer

Figure 5. An example structure of BP network.

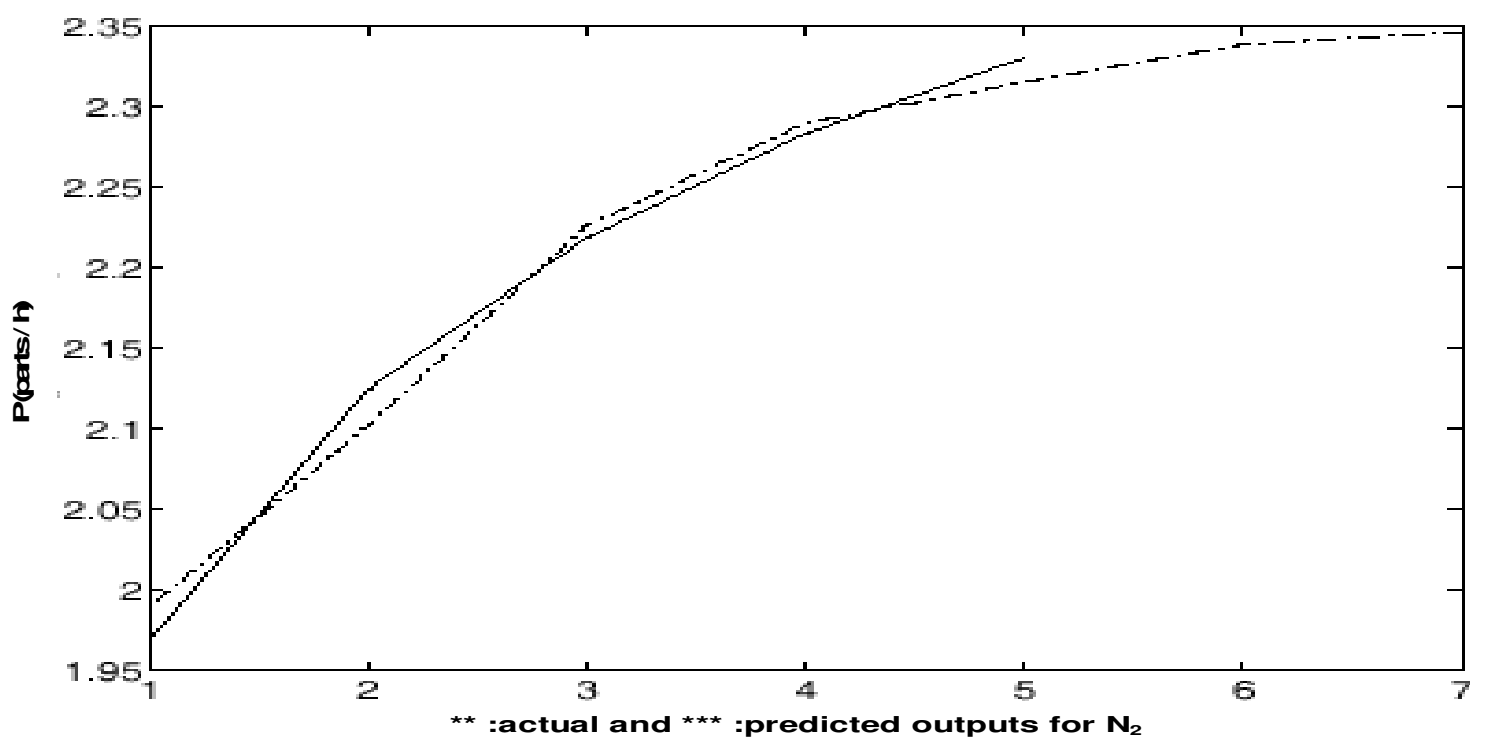

Figure 6. Actual and predicted outputs for $\mathrm{N}_{2}$ in case 1 .

useful as a rapid reanalysis tool to predict the production rate of the transferline, by using prior information instead of performing reanalysis of the whole system. As a future study, recurrent neural networks may be studied for the same purpose and for the analysis of the queuing systems.

\section{REFERENCES}

Al-jaar RY, Desrochers AA (1989). Petri Nets in Automation and Manufacturing. Advances in Automation and Robotics, JAI Press, 2: 57-75.
Arslan MA (1993). Data Clustering and Weight Analysis Enhancements to the Back Propagation Neural network. Master Thesis, RPI, Troy, NY.

Berke L, Hajela P (1992). Applications of Artificial Neural Networks to Structural Mechanics. J. Struct. Optim., 3(1) 35-42.

Dariavach N, Liang J, Barr G, Shangguan D (2010). Effects of multiple BGA rework on strength of solder joints. Soldering \& Surface Mount Technol., 22(4): 20-30.

Desrochers AA (1990). Modelling and Control of Automated Manufacturing Systems. IEEE Computer Society Press.

Dubois D, Stecke KE (1982). Using Petri Nets to represent Production Process. Proceedings of the 22nd IEEE Conference on Decision and Control, San Antonio, Texas,. pp.1062-1067.

Fidan I (2004). CAPP for Electronics Manufacturing - Case Study: Fine Pitch SMT Laser Soldering. Transactions of the ASME-J. Elect. 
Pack., 126(1):173-176.

Fidan I, Roush EM, Tumkor S, Kraft RP (2004). Intelligent Simulation Environment for Electronics Remanufacturing Systems. Proceedings of the 29th IEEE/CPMT/SEMI Int.ernational Electron.ics Man.ufacturing Technol.ogy Symposium., pp.160-164.

Fidan I, Roush Em, Tumkor S, Kraft RP (2006). Internet Based Electronics Manufacturing Troubleshooting Tool for Surface Mount PCB Assembly. Int. J.Adv. Manuf. Technol., 27(1): 561-567.

Fu B, Hajela P (1992). An Introduction to Anolog Computing With Artificial Neural Networks. Rensselaer Polytechnic Institute, Troy, NY.

Hajela P, Szewczyk Z (1992). On the use of ANN Interconnection Weights in Optimal Structural Design. Proceedings of the 4th AIAA/NASA/Air Force Symposium, Cleveland, Ohio.
Rosenblatt F (1962). Principles of Neurodynamics. Spartan-NewYork. Vendan SP, Sakthidhasan K (2010). Reduction of Wastages in Motor Manufacturing Industry. J. Mech. Industr. Eng., 4(5): 579-590. 